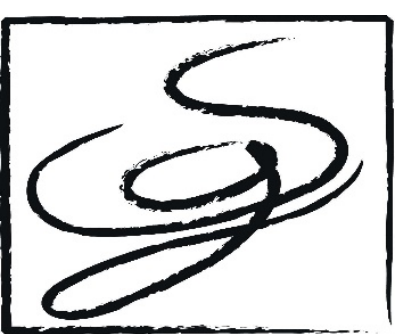

SEMIMARIO DE INUESTIGACIÓN DE GÉNERO Y ESTUDIOS GULTURALES

\section{Los roles de género y la identidad de las mujeres chicanas/ latinas en Loverboys de Ana Castillo}

\author{
Gender roles and identity in the Chicana/Latina \\ Women in Loverboys by Ana Castillo
}

\author{
María de la O Merino Aguilera \\ Hochschule Stralsund \\ merino.mariola@gmail.com
}

Fecha de recepción: 29/09/2021 Fecha de evaluación: 25/10/2021

Fecha de aceptación: 11/11/2021

\title{
Resumen
}

Ana Castillo (Chicago, 1953) es la autora de la colección de relatos Loverboys (1996). Estos relatos abordan la realidad de las mujeres chicanas y latinas en los EE. UU. a partir de una pluralidad de contextos sociales. El propósito de este artículo es analizar los relatos de Loverboys, desde la perspectiva de los roles de género y el concepto de identidad, así como presentar una breve observación inicial sobre cómo las voces femeninas han sido silenciadas a lo largo de la historia. La hipótesis que se desea demostrar es que Castillo defiende, conserva y pretende restaurar en estos relatos los valores de las mujeres chicanas y latinas, repetidamente denostados por el machismo de la sociedad patriarcal, a través de los personajes femeninos de los relatos recogidos en Loverboys.

Palabras clave: Ana Castillo; Loverboys; roles de género; identidad, chicanas; latinas.

\section{Abstract}

Ana Castillo (Chicago, 1953) is the author of the short story collection Loverboys (1996). These stories address the reality of Chicana and Latina women in the U.S. from a plurality of social contexts. The purpose of this article is to analyse the stories in Loverboys from the perspective of gender roles and the concept of identity, as well as to present a brief initial observation on how female voices have been silenced throughout history. The hypothesis to be demonstrated is that, in these stories, Castillo defends, preserves, and seeks to restore the values of Chicana and Latina 
women, repeatedly reviled by the machismo of patriarchal society, through the female characters in the stories collected in Loverboys.

Key words: Ana Castillo; Loverboys; gender roles; identity; Chicana; Latina.

\section{Introducción}

La colección de relatos de Ana Castillo, Loverboys, está cohesionada por un tema común que se repite en las diferentes historias: el deseo, en mayor parte en su variante sexual. A través de este leit motif, Castillo desnaturaliza los componentes culturales e históricos de sus personajes femeninos al destruir los estereotipos literarios sobre la mujer, al tiempo que devuelve el poder al papel de la mujer chicana y latina en su texto. En palabras de B. Milligan, en el prólogo a su entrevista con Castillo:

Castillo has become one of the most outspoken voices for the "colonized woman" and has taken brave positions opposing the suppression of "brown women" in an elitist, racist society that generally acknowledges race relations as pertaining only to black and white divisions (Milligan, 1999: 20).

Castillo en Loverboys debate varias cuestiones centrales en la crítica feminista: los conceptos de historia y escritura; aspectos sexuales y de género y por último, la cuestión de la identidad, como la oposición del yo frente al otro.

En una primera parte, se presenta, en términos generales, la interrelación existente entre la escritura y el silencio de las voces femeninas. Es por todos sabido que, lo largo de la historia, las voces femeninas han sido acalladas y estereotipadas en la literatura y en otras expresiones artísticas. Pocas eran las mujeres que podían o tenían permitido escribir, y por ende, hablar de lo que a ellas les afectaba o les apelaba.

En un segundo apartado, se discute cómo los hombres han creado a lo largo de los siglos un discurso sexual hegemónico, en el cual la mujer era un objeto y un mito, con dos representaciones opuestas, la puta o la virgen.

Para finalizar, se plantea el concepto de identidad: el yo en relación con el otro. Es difícil considerar al yo como algo aislado, es el otro quién le da sentido y lo define en términos relacionales. En palabras de Gloria Anzaldúa, decir de alguien "you are nothing but a woman' means you are defective" (Anzaldúa: 1987, 105). En este apartado, se analiza cómo Castillo rechaza esta idea y la revierte en los relatos que conforman Loverboys, con la intención de empoderar a los personajes femeninos de los mismos. 


\section{Historia y escritura}

Para la teoría feminista, el discurso de la razón ha suprimido las voces marginales, aquellas que no encajan dentro del modelo blanco, masculino y heterosexual dominante. La crítica feminista Hélène Cixous defiende que una de las formas más significativas de ejercer poder en las sociedades patriarcales de occidente ha sido el discurso literario. La «escritura marcada», usando el término de Cixous, silenciaba la experiencia de las mujeres, entre otros grupos marginales (como las minorías raciales y de identidad sexual) al presentar un estereotipo sancionado por la tradición patriarcal.

Este estereotipo es conocido como el "eterno femenino», la mujer ideal. Annie O. Eysturoy, en su obra crítica Daughters of Self-Creation: The Contemporary Chicana Novel explica cómo este concepto del eterno femenino fue fomentado por numerosos autores del siglo XIX, Goethe entre ellos. Parafraseando a Eysturoy, el eterno femenino según Goethe es la mujer ideal, un modelo de abnegación y pureza de corazón, la cual lleva una vida de pura contemplación, una vida que no puede ser contada, pues no hay historia. Para Goethe, las mujeres son simples recipientes, vacías de fuerza creadora. De este modo, niega a las mujeres su capacidad creativa, tanto en el campo de la escritura como en otras áreas artísticas, y ratifica así su silencio.

Según Eysturoy, la presión de este modelo de mujer ideal se hace aún más evidente en la mujer chicana:

For the Chicana, twice a minority, the silence has been especially deep: constricted simultaneously by patriarchal cultures and the dominant cultural stereotypes of her Chicana identity, the Chicana has been defined and confined within the mother/ virgin/ whore stereotypes of the past, both within her own Chicano culture and the larger American cultural context" (Eysturoy, 1996: 24).

En el relato de Castillo «Conversations with an Absent Lover on a Beachless Afternoon» dentro de la colección Loverboys, la voz narrativa niega este modelo ideal femenino: «Women are no angels. Oh, we are no innocents» (Castillo, 1996: 152). Castillo se enfrenta a la concepción de la mujer como un ángel, alguien pasivo que esta exento de capacidad creativa, como leíamos en la descripción del concepto de eterno femenino de Goethe. Así, algunas de las figuras femeninas en los relatos de Castillo son artistas, profesoras, actrices; en otras palabras, ocupan un lugar propio en el mundo profesional, lo que se traduce también en el ámbito personal, puesto que estos personajes femeninos también toman las riendas de sus relaciones amorosas, abandonando la pasividad igualmente en esta área de sus vidas.

Para volver a Cixous, su propuesta es contrarrestar la represión ejercida históricamente por la escritura marcada subvirtiendo las propias nociones del discurso patriarcal. Al ser la propia escritura el instrumento para silenciar las voces marginales es, por tanto, a su vez el medio 
apropiado para posibilitar la expresión de la mujer. De esta forma, Castillo en los relatos de Loverboys centra las diferentes historias en la marginalidad de la experiencia de las mujeres chicanas y latinas. En algunos casos las historias de estas mujeres tienen una sexualidad abiertamente en contra de la norma sancionada por la comunidad, es decir, llevan las riendas de su vida sexual, lo que en sus comunidades está restringido a los hombres, como en el relato "Vatolandia», en «Juan in a Million» o en "María Who Paints and Who Bore José Two Children». Así, Castillo visibiliza las historias de vidas de mujeres que han sido ignoradas históricamente por el discurso dominante, ya sea este el del hombre anglo o el chicano. De este modo, Castillo ofrece con su narrativa una resistencia al

Las historias narradas en Loverboys se alejan de normas sancionadoras; Castillo nos acerca en sus relatos a la experiencia plural de la mujer chicana. Las protagonistas viven circunstancias diferentes, tanto económicas como personales. Es así como se niega toda posibilidad de una cualidad homogénea en la categoría "chicana», oponiendo la multiplicidad de opciones y planteamientos a la idea de un texto totalizador y estandardizado. En los relatos "Loverboys» o "Vatolandia», las protagonistas encarnan a mujeres de éxito en sus diferentes profesiones, mujeres independientes que controlan sus destinos. Por otro lado, en «My Dream Last Night» o en "Ghost Talk», se relatan las historias sórdidas de abusos a mujeres chicanas y la invisibilidad de la que estas son objeto frente a la sociedad norteamericana.

Estos dos polos opuestos, la cara y la cruz de la experiencia de la mujer chicana y latina en los EE.UU. hoy, crean el marco para las distintas historias, en las cuales la situación de las protagonistas varía en función de las diferentes circunstancias personales de cada personaje. La pobreza de la familia en la sórdida historia "Christmas Story of the Golden Cockroach» contrasta con la frivolidad y el modo de vida de la protagonista en «Juan in a Million», quien lleva una doble relación amorosa, vive en un edificio con portero privado, y puede permitirse un viaje alrededor del mundo. Los protagonistas del relato «If Not for the Blessing of a Son», emigrantes políticos acomodados, tienen a su vez una situación personal y una actitud diametralmente opuesta a la protagonista de «Mother's Wish», en cuanto a la relación con sus hijos. En el relato «If Not for the Blessing of a Son», los padres prácticamente hacen de su propio hijo un esclavo, cargándolo con una lista interminable de tareas domésticas, algo que por otra parte «as a good Catholic son it would have been his duty nonetheless» (Castillo, 1996: 36). En «Mother's Wish», por otra parte, vemos como la relación maternofilial es la contraria: en este brevísimo relato, el hijo es casi idolatrado por su madre, hasta el punto en que se afirma que este no morirá nunca, asumiendo así la imagen divina que tiene el hijo para la madre.

El relato "Ghost Story» nos presenta una realidad muy distinta de la narrada en el relato «A True Story», aunque el deseo es el motor de ambas historias. En "Ghost Story» la madre de la protagonista sufre abusos sexuales por parte de su patrón, lo cual desencadena un deseo de venganza en su hija, mientras que el deseo en «A True Story» es 
abiertamente sexual por parte de la voz narrativa hacia la mujer que tiene delante. El cliché de la imagen que ha existido tradicionalmente de la familia chicana cargada de hijos, con una vida miserable en los EE. UU., es de este modo rechazada por Castillo, al ofrecer una pluralidad de personajes con circunstancias vitales diferentes. En definitiva, la experiencia latina / chicana no es única; del mismo modo que no hay dos vidas idénticas, no puede haber dos historias iguales.

\section{Los roles sexuales y de género}

Un aspecto de la ruptura de los relatos con el modelo clásico patriarcal es el estilo narrativo de los mismos. En oposición a una organización lineal, basada en los principios de causa y efecto, muchas de las historias están repletas de comentarios y digresiones, las cuales ayudan a crear una atmósfera similar a la de las narraciones orales, más relacionadas con las sociedades matriarcales. Un ejemplo de esto lo hallamos en el uso frecuente de preguntas retóricas en varios de los relatos como «Loverboys» y «Who Was Juana Gallo?», entre otros. En estos relatos, la voz narrativa se dirige a un lector imaginario de forma directa: en "Loverboys», la narradora alude al lector cuando afirma «He's really gonna hate me for telling you all this...» (Catillo, 1996:15), del mismo modo que en «Who was Juana Gallo», cuando la voz narrativa se dirige al lector: «But forgive my digression and let me tell you about Juana Gallo, as I promised» (Castillo, 1996: 26) Este recurso contribuye a su vez a la creación de un lector implícito que asume el papel de destinatario de las confesiones de los narradores.

De este modo, el discurso de la razón es desplazado, y el deseo y los sentimientos ocupan un lugar central en la narración. La sentimentalidad femenina adquiere una visión muy heterodoxa en los relatos que conforman Loverboys. Las protagonistas son en su mayoría mujeres independientes que no se adscriben a una única opción o a un único objeto amoroso. Se rechaza la victimización y la dependencia con la misma fuerza que se denuncia el machismo y la guetoización dentro de la propia comunidad étnica. Esta denuncia del machismo, que está presente en la mayoría de los relatos, se hace especialmente patente en el relato "Conversations with an Absent Lover on a Beachless Afternoon»:

Your mother was locked up in her room on her wedding night
by her husband while he went out carousing. [...] My sister
was beaten on the street on her wedding night by her
groom, the man to whom she has been married now for
twenty-five years. His whole family looked on. [...] My
cousin has had seven children. She is not allowed to have
visitors. She does not have a telephone. Her husband has
kept her hidden for nearly twenty years (Castillo, 1996: 158).

Según M. M. Mowad, en su disertación «Machismo and the border challenges to ideology and Chicano/a gender roles», la ideología del machismos «has been described as both a cult and a code of masculinity for Latino men. The word macho is derived from the Aztec word which 
means the image or reflection of myself» (Mowad, 2004: 3). Para Mowad, Castillo en Massacre of the Dreamers afirma que el machismo emerge para reforzar la hegemonía masculina, donde la mujer se encuentra en una posición de subordinación y dependencia para su "bienestar». Evidentemente, este «bienestar" es susceptible en muchos casos de ser cuestionado, e incluso denunciado, en muchos relatos de Loverboys, cuando salen a la luz el maltrato machista que sufren algunos personajes femeninos a manos de sus parejas o sus patrones, como vemos en «Ghost Talk» donde la madre de la protagonista es violada por su jefe y denigrada: "Who was she, but some Mexican whore...» (Castillo, 1996: 51) o en el relato «My Dream Last Night»: «The man there, where Mirna went, began bothering Mirna. She was too afraid to tell anyone» (Castillo, 1996: 103).

El simbolismo es también un recurso en varios de los relatos, un elemento íntimamente relacionado por la crítica feminista con la escritura hecha por mujeres. En el primer relato que da título a toda la colección, "Loverboys», encontramos un ejemplo del simbolismo anteriormente mencionado. En la librería, el chico pregunta por una copia de la novela El Rebelde, de Camus. En lugar de comprarlo, finalmente se decide por otro libro del mismo autor El Extranjero (otra posible traducción del título en inglés sería «el extraño»). Los títulos de ambos libros son alegóricos: la narradora resultará ser una rebelde para su joven amante, una mujer demasiado activa y autosuficiente para la familia y las expectativas de éste. Al mismo tiempo, el chico es en realidad un extraño para la narradora, quien no conoce nada con certeza de él: «He likes poetry. He writes poetry. At least he says he does. He never showed me anything» (Castillo, 1996: 14). El desconocimiento casi total de su amante por parte de la protagonista es dolorosamente obvio cuando esta intenta sin éxito contactar con él tras su ruptura. Así, el paralelismo entre el primer encuentro en la librería y el fin de la historia de amor de ambos en términos simbólicos se hace patente.

Otra denuncia del discurso dominante y un arma para devolver la voz a la experiencia marginal chicana es el uso constante de juegos de palabras o palabras con doble sentido en inglés y español en varios de los relatos, conformando así un texto híbrido. Al reclamar una voz para la mujer chicana, esta voz no puede ser distinta al reflejo de la realidad de la vida en la frontera, usando la expresión de Anzaldúa, en el que la mezcla de lenguas está de continuo presente. La hibridación textual es un palimpsesto fruto de la mezcla y el encuentro de las culturas presentes en la experiencia chicana y latina, al margen de un lenguaje puro, sin mestizaje. Del mismo modo, Castillo recurre a aspectos autobiográficos en sus relatos (como se hace evidente en el relato "Subtitles») que se mezclan con historias de vidas ajenas, las cuales en muchos casos reflejan situaciones cotidianas de la realidad chicana y latina, dando lugar a un patchwork de textos que contribuyen así a su naturaleza híbrida.

Los roles sexuales y de género también son subvertidos en Loverboys, y el discurso sentimental deconstruido se aleja de la norma hetero y se ofrece en sus múltiples permutaciones. Encontramos relaciones homosexuales, bisexuales, heterosexuales y platónicas, entre otras. No sólo la orientación sexual de los amantes es iconoclasta, también sus 
actitudes lo son. Con las protagonistas de historias como "Vatolandia», «Loverboys», "Juan in a Million» o «Again, Like Before», Castillo muestra cómo el concepto de mujer no es en sí esencialmente un concepto determinado $y$, dado el factor cultural que influye en los sujetos para la percepción de las identidades, concluye que los roles de género pueden ser intercambiados, en tanto que provisionales e inestables. Así en «Vatolandia», entre otros relatos, la protagonista clasifica a sus amantes asumiendo un papel típicamente masculino y rechazando una actitud pasiva y sumisa en sus relaciones con los hombres. En palabras de Katherine Sugg, Castillo persiste. como otras escritoras chicanas en demostrar la profunda relación entre las identidades étnico-raciales y las identidades disciplinarias de la sexualidad y el género (Sugg: 2003, 139).

De esta forma, muchos de los personajes femeninos de las historias desean ser protagonistas de sus vidas, como María en «María Who Paints and Who Bore José Two Children». Al hacer esto, corren el riesgo, como María, de ser acusadas de egoísmo, ya que con su actitud se rebelan contra el patrón idealizado de la mujer contemplativa. La decisión de María de realizarse profesionalmente es paralela a su relación sentimental con una mujer. Se trata de una liberación sexual y personal en la vida de una madre y esposa sacrificada, víctima de un matrimonio vacío y sin amor.

En el relato "Juan in a Million», la protagonista adopta un rol masculino igualmente, al mantener relaciones simultáneas con diferentes hombres en una inversión del clásico papel donjuanesco. El hecho de que las narradoras femeninas asuman roles típicamente representativos de hombres es un intento claro de deconstruir conceptos esencialistas y desnaturalizar los roles de género. Al asumir estos papeles tradicionalmente masculinos, las protagonistas pasan de ser modelos ideales para ser catalogadas como monstruos. La dicotomía virgen/ puta expuesta por Eysturoy se relaciona así con la pérdida de la inocencia, el concepto tradicional del "ángel doméstico» se contrapone con el de la mujer independiente, que expresa sus ideas y aún más, se atreve a escribirlas. Una muestra la encontramos en el relato "Conversations with an Absent Lover on a Beachless Afternoon»: « [...] and now, what would he think of the monster that I had become? And the thoughts that I have. The godless, female thoughts I have. And speak. And write» (Castillo, 1996: 153-4). Por otra parte, En el relato «A Foreign Market», la protagonista es tomada por una prostituta solo porque responde activamente a su deseo sexual, sin actuar de forma hipócrita y recatada en su primer encuentro con un total desconocido. Cuando los personajes femeninos pierden su aura de inocencia, sus compañeros a su vez pierden todo el interés hacia ellas. El tema de la falsa inocencia femenina es denunciado, así, en este y otros relatos.

La protagonista de "Conversations with an Absent Lover on a Beachless Afternoon» es víctima de esta situación con el padre de su hijo:

Months before, I had lost all pretences to innocence. I didn't know then that perceiving me innocent was what the 'father' had loved about me. Later, not because the innocence was gone or had ever been there, but simply when he 
realized I was not innocent, he began to be cruel to me $\quad[\ldots]$ I didn't know that my sin, my betrayal was that I had dared to love him in return (Castillo, 1996: 150).

Al actuar de forma activa en la relación, la protagonista de este relato, al igual que las protagonistas de "Foreign Market» o "Vatolandia", pierde cualquier atisbo de inocencia y se equipara sexualmente con su pareja, adoptando así una actitud amenazadora para el personaje, que presenta claramente una actitud machista. En palabras de Anzaldúa:

The culture and the Church insist that women are subservient to males. If a woman rebels she is a mujer mala. If a woman doesn't renounce herself in favor of the male, she is selfish. If a woman remains a virgen until she marries,she is a good woman (Anzaldúa, 1987: 39).

Por tanto, las protagonistas de muchos de los relatos de Loverboys tienen que luchar contra la conceptualización de mujer como "el ángel de la casa" para conseguir su realización personal y su liberación tanto en términos sexuales como culturales. Para B. Evenson, en Loverboys, la sexualidad es contemplada como algo fluido: "Sometimes this is echoed culturally when characters seem to experience similar fluidity in terms of possessing a social identity that makes multiple claims on the individual» (Evenson, 1997)

\section{La identidad}

Por último, en Loverboys muchas de sus protagonistas se hallan envueltas en procesos más o menos implícitos de desarrollo personal y autoconocimiento. Se podría así decir que varios de los relatos se centran en el tema de la búsqueda de la identidad, pues, en palabras de la propia Castillo en la entrevista con Milligan, «if you don't know who you are and if what you do know has been brought to you in such a manner that you feel bad about it, this will perpetuate the same images» (Milligan, 1999: 25). En este proceso de búsqueda de sí mismas, las narradoras se encuentran con definiciones culturales y sociales que las delimitan y constriñen. Citando a Roland Walter en The Cultural Politics of Dislocation and Relocation in the Novels of Ana Castillo:

Castillo's characters, male and female, are border subjects positioned between cultures and in search of an alternative to their lived 'nepantla' state of invisibility and transition. In terms of her female characters, this state is aggravated by what Castillo calls in Massacre_of the Dreamers 'double-sexism, being female and indigenous'. (Walter, 1998:

82)

La opción de narrar su propia historia es en sí un acto de rebelión contra el discurso dominante. Cada historia es un intento de individualizar la experiencia chicana, en una polifonía de voces de mujeres muy frecuentes dentro de la técnica feminista. Al mismo tiempo, es un acto de denuncia de los esencialismos, de la identidad colectiva, a la vez que un 
intento de devolver la voz tanto tiempo silenciada. Castillo, en la entrevista anteriormente mencionada de Milligan, afirma que, como escritora «l do know that I am on a mission as far as asserting my identity in this society that, as I say, doesn't recognize you unless you are white or black» (Milligan, 1999: 22).

El papel de la memoria en la construcción del sujeto adquiere aquí una importancia esencial. Según A. D. Juan Rubio e I. García Conesa en su artículo "La Figura de Ana Castillo en la Literatura Chicana Femenina», en la literatura chicana escrita por mujeres «emerge la salvaguardia de la tradición oral y la memoria» (Juan Rubio y García Conesa, 2018: 103). La narración cargada de deseo y basada en el recuerdo es un elemento que individualiza y personaliza, como en el relato «Who Was Juana Gallo?». En él, la voz narradora intenta delinear la verdadera identidad de Juana, utilizando para ello su deseo hacia ella como catalizador de sus recuerdos, interpelando a un lector/confidente en su discurso.

En «Ghost Talk» es quizá el relato donde más claramente se perciba la marginalidad de la narradora como mujer indígena en términos del doble sexismo de Walter: «We are but we do not speak» (Castillo, 1996: 45). Aquí el deseo tiene un aspecto claramente distinto al sexual. La narradora busca justicia, el suyo es un deseo de venganza frente a la situación de opresión de su madre y de tantas mujeres chicanas que, como ella, han sido objeto del abuso de hombres sin escrúpulos, en su mayoría anglos. De este modo, la voz narradora realiza la función que Juan Rubio y García Conesa identifica como propia en las escritoras chicanas: «Las escritoras chicanas reexaminan, cuestionan, subvierten y refuerzan los valores de la sociedad patriarcal. Sus narrativas son manifestaciones de las luchas contra múltiples formas de opresión» (Juan Rubio y García Conesa, 2018: 103). Pues, según Anzaldúa:

there in front of us is the crossroads and choice: to feel a victim where someone else is in control and therefore responsible and to blame (...) or to feel strong, and, for the most part, in control: (Anzaldúa, 1987: 43).

Por otra parte, no es extraño que algunas de las protagonistas de los relatos sean mujeres creativas artísticamente («Loverboys», "María Who Paints and Who Bore José Two Children», "Conversations with an Absent Lover on a Beachless Afternoon»). En el proceso de búsqueda personal, la expresión artística, el derecho de la auto representación, es una forma de controlar y modificar el entorno circundante, es lo contrario a la mujer dominada y recluida en la casa.

El hecho de que el relato de la curandera aparezca en último lugar tampoco es accidental. En términos simbólicos podría representar la apuesta de Castillo por la participación activa de la mujer chicana en la creación de su historia, en su propia sanación. En contra de la connotación negativa que la figura de la bruja ha recibido tradicionalmente, Ana Castillo reivindica el papel de la bruja o curandera como una mujer con unas características especiales:

However, I claim this term (bruja) for women who are in tune with their psyches, allow their lives to be informed by them, 
and offer their intuitive gifts to their communities without fear of being seen as loathsome or mad. [...] lf we dispense with that fear but retain or reinstate our insights and connections with all living things, we have a woman with developed psychic resources, a bruja" (Castillo, 1994: 157)

Por otra parte, T. Voronchenko afirma que Gaspar de Alba «compares Chicana writers to curanderas and brujas because they are keepers of the culture, the memories, the rituals, the stories, the superstitions, the language and the imagery of the Mexican Heritage» (Voronchenko, 2014: 81). De este modo, el proceso poético es en sí mismo considerado como sanador para la comunidad, pues preserva su tradición. El papel de la escritora chicana y de la curandera es, por tanto, un papel político: la defensa, la conservación y la restauración de los valores chicanos y el de sus individuos como colectividad.

\section{Conclusión}

La colección de relatos Loverboys escenifica las historias tristes y divertidas, duras y esperanzadoras de las mujeres chicanas y latinas en búsqueda de un lugar y una voz propios fuera de los márgenes de la sociedad norteamericana. Una voz que ha sido doblemente silenciada, en la comunidad anglo y en la propia comunidad chicana/latina, de carácter marcadamente machista. Castillo, en la colección de relatos Loverboys, denuncia este silenciamiento, a la vez que nos presenta una pluralidad de historias que reflejan las realidades igualmente plurales de las mujeres chicanas en los EE. UU. Castillo defiende, conserva y pretende restaurar en sus relatos los valores de las chicanas, repetidamente denostados por el machismo de la sociedad imperante. Loverboys es así una reflexión sobre la situación de las mujeres chicanas en los años 90 y una forma de reivindicar la reapropiación de su pasado, el papel activo en su presente y la apuesta por su futuro.

\section{Referencias bibliográficas}

ANZALDÚA, Gloria. Borderlands/La Frontera. San Francisco: Aunt Lute Books, 1987.

CASTILLO, Ana. Loverboys. Nueva York: Plume, 1996.

CASTILLO, Ana. Massacre of the Dreamers. New York: Penguin, 1994.

CIXOUS, Hèléne, Keith Cohen y Paula Cohen. The Laugh of the Medusa. Signs, Vol. 1, No. 4 (Summer, 1976), pp. 875-893. The University of Chicago Press. http://www.jstor.org/stable/3173239

EVENSON, Brian (1997, Spring). Loverboys. Review of Contemporary Fiction, 17, 201.

https://www.proquest.com/magazines/loverboys/docview/235919426/se2?accountid $=14695$

EYSTUROY, Annie Olivia. Daughters of Self-Creation. New Mexico: University of New Mexico, 1996. 
JUAN RUBIO, Antonio Daniel e Isabel María García Conesa. "La figura de Ana Castillo como representante de la literatura chicana femenina" Revista Internacional de Culturas y Literaturas, Universidad de Sevilla, junio 2018.

MILLIGAN, Bryce. "An Interview with Ana Castillo." <i>South Central Review</i>, vol. 16, no. 1, 1999, pp. 19-29. <i>JSTOR</i>, www.jstor.org/stable/3189709

MOWAD, Monique Mary. (2007). Machismo and the border challenges to ideology and Chicano/a gender roles (Order No. 1448901). Available from ProQuest Dissertations \& Theses A\&l; ProQuest Dissertations \& Theses Global. (304713631). https://www.proquest.com/dissertations-theses/machismoborder-challenges-ideology-chicano/docview/304713631/se2?accountid $=14695$

SUGG, Katherine. "The Ultimate Rebellion: Chicana Narratives of Sexuality and Community." Meridians, vol. 3, no. 2, Indiana University Press, 2003, pp. 13970, http://www.jstor.org/stable/40338579

VORONCHENKO, Tatiana. "American Studies in Russia: Learning Chicana/o Literature in Chita (Siberia)". International Perspectives on Chicana/o Literature: This World is my Place. Ed. C. Leen and N. Thornton. New York: Routledge, 2014.

WALTER, Roland. The Cultural Politics of Dislocation and Relocation in the Novels of Ana Castillo. MELUS, Spring, 1998, Vol. 23, No. 1, Latino/a Literature (Spring, 1998), pp. 81- 97 Oxford University Press. https://www.jstor.org/stable/467765 